\section{Favorable event free-survival of high-dose chemotherapy followed by autologous hematopoietic stem cell transplantation for higher risk diffuse large B-cell lymphoma in first complete remission}

\author{
Hiroto Kaneko, ${ }^{1}$ Yasuhiko Tsutsumi, ${ }^{1}$ \\ Takahiro Fujino, ${ }^{1}$ Saeko Kuwahara, ${ }^{1}$ \\ Muneo Ohshiro, ${ }^{1}$ Toshiki Iwai, ${ }^{1}$ \\ Junya Kuroda, ${ }^{2}$ Shouhei Yokota, ${ }^{2}$ \\ Shigeo Horiike, ${ }^{2}$ Masafumi Taniwaki ${ }^{2}$ \\ 1'Department of Hematology, Japanese \\ Red Cross Kyoto Daiichi Hospital; \\ 2Department of Hematology/Oncology, \\ Kyoto Prefectural University of Medicine, \\ Japan
}

\begin{abstract}
High-dose chemotherapy followed by autologous stem cell transplantation (ASCT) has been applied to patients with diffuse large Bcell lymphoma (DLBCL); it is well established that ASCT shows significant survival benefits for chemosensitive relapse. However, half of relapsed patients are resistant to salvage chemotherapy, indicating that they are not suitable for ASCT. We retrospectively analyzed the clinical records of 47 patients with DLBCL classified as high or high-intermediate (higher) risk, according to the International Prognostic Index, who underwent upfront ASCT in first complete remission (CR1). Compared with 10 patients with similar characteristics who did not receive ASCT, event free survival at 5 -year was significantly superior in ASCT group. Toxicity of ASCT was acceptable and therapy-related death was not observed. We therefore propose that upfront ASCT for higher risk DLBCL in CR1 might provide survival benefit, probably because the high-dose therapy removes minimally resided tumor.
\end{abstract}

\section{Introduction}

Diffuse large B-cell lymphoma (DLBCL) is the most frequent aggressive lymphoma in Japan. Although the majority of patients with DLBCL achieve complete remission (CR), about $40 \%$ of them die of the disease. ${ }^{1}$ This suggests that minimal residual tumor, that is not detected in clinical CR state, induces relapse. International Prognostic Index (IPI or age-adjusted IPI) is considered the most valuable prognostic indicator of aggressive lymphoma. ${ }^{2,3}$ Prognosis of patients classified as high or high-intermediate (higher) risk by IPI is extremely poor, mainly because of the high relapse rate. In order to improve the prognosis of higher risk patients, undetectable residual tumor in first CR (CR1) state should be removed to prohibit relapse.

High-dose chemotherapy followed by autologous stem cell transplantation (ASCT) has been applied to patients with DLBCL in a number of cases. ${ }^{4-14}$ Although it is well established that ASCT shows significant survival benefit for chemosensitive relapse of aggressive lymphoma, ${ }^{15}$ half of relapsed patients are actually resistant to salvage chemotherapy, resulting in dropout from ASCT. ${ }^{16}$ Haioun et al. reported that only 21 out of 59 patients who relapsed could receive salvage ASCT because of refractoriness to chemotherapy. ${ }^{8}$ Other studies also showed extremely poor prognosis of relapsed aggressive lymphoma despite various salvage therapy, ${ }^{17,18}$ indicating that most of relapsed patients are not suitable for ASCT.

We therefore conclude that the reduction of relapse rate of higher risk DLBCL in CR1 might lead to a better prognosis and we retrospectively analyze clinical outcome of upfront ASCT for those patients.

\section{Materials and Methods}

Between April 1997 and March 2014, we treated 224 patients with DLBCL (age <70); among them, 102 were classified as high or high-intermediate risk by IPI (or age-adjusted IPI). ${ }^{2,3}$ They were considered as candidates for upfront ASCT when CR, as per International Workshop Criteria, ${ }^{19}$ or in combination with fluorine-18-fluorodeoxyglucose positron emission tomography, ${ }^{20}$ was achieved within six courses of $\mathrm{CHOP}$ or rituximab-combined (R)CHOP regimen. ${ }^{21,22}$ Eligibility criteria were: ejection fraction of $50 \%$ (or more) on ultrasound cardiographs, serum creatinine less than $2 \mathrm{mg} / \mathrm{dL}$, total bilirubin less than $3 \mathrm{mg} / \mathrm{dL}$, absence of infections as human immunodeficiency virus or hepatitis B virus, and performance status by Eastern Cooperative Oncology Group 2 or less. Stem cells were harvested with mobilization therapy of high-dose etoposide (500 mg per square on days 1 to 3 ). After acquiring written informed consent, high-dose chemotherapy of MCVC regimen was administered. The regimen consisted of ranimustine $200 \mathrm{mg}$ per square on day -8 and -3 , carboplatine $300 \mathrm{mg}$ per square on days -7 through -4 , VP16 $500 \mathrm{mg}$ per square on days -6 to -4 , and cyclophosphamide $50 \mathrm{mg}$ per $\mathrm{kg}$ on days -3 and -2 . On day 0 , ASCT was carried out.

Regimen related toxicity (RRT) was record-
Correspondence: Hiroto Kaneko, Department of Hematology, Japanese Red Cross Kyoto Daiichi Hospital, 15-749 Honmachi, Higashiyama-ku, Kyoto 605-0981, Japan.

Tel.: +81.7556.11121 - Fax: +81.7556 .16308 .

E-mail: hiroto-kaneko@kyoto1-jrc.org

Key words: Diffuse large B-cell lymphoma; autologous transplantation; upfront.

Contributions: HK, JK, SH, designed this study and MT supervised it; HK, YS, TF, SK, MO, and TI have treated the patients included in the study; HK and SY analyzed clinical data; HK wrote the manuscript and MT critically revised it.

Conflict of interest: the authors declare no potential conflict of interest.

Received for publication: 14 January 2015.

Accepted for publication: 16 April 2015.

This work is licensed under a Creative Commons Attribution NonCommercial 3.0 License (CC BYNC 3.0).

(C) Copyright H. Kaneko et al., 2015

Licensee PAGEPress, Italy

Hematology Reports 2015; 7:5812

doi:10.4081/hr.2015.5812

ed according to Common Terminology Criteria for Adverse Events v4.0. Re-growth of remitted lesion or appearance of new lesion was defined as relapse. Survival time was calculated from ASCT to death; any cause or relapse was regarded as event. Using SPSS version 19.0, survival rates were estimated by KaplanMeier method and compared by log-rank test.

\section{Results}

Seventy-five patients (73.5\%) achieved CR1. Among these, relapse before ASCT occurred in 14 patients, severe icterus due to liver invasion was observed in one patient, performance status progressed into $3-4$ in 3 patients, and 10 patients rejected ASCT. Consequently, only 47 patients in CR1 underwent ASCT. Their characteristics are summarized in Table 1 . Histological subtypes were CD5-positive for DLBCL in 7 patients, germinal center B-cell (GCB) type in 7, and non-GCB type in 16 . Unfortunately, the subtype of the remaining 17 patients was not determined. $\mathrm{R}$ was used in combination with induction, mobilization, and high-dose therapy in 19 patients; 0.7$27.6 \times 10^{6} / \mathrm{kg}$ of CD34-positive cells were infused (median 6.9). No graft failure was observed. The day of neutrophil recovery over $500 / \mathrm{mm}^{3}$ ranged from 7 to 14 (median 9) and platelet over $2 \times 10^{4} / \mathrm{mm}^{3}$ from 7 to 26 (median 
11.5). Median units of transfusion of red blood cells and platelet were 0 (range 0-8) and 20 (060 ), respectively. Life-threatening RRT was sepsis of methicillin-resistant Staphylococcus aureus complicated in a patient (2.1\%) (Table 2 ), while the most common one was febrile neutropenia that occurred in 35 patients (74.5\%). Non-hematological RRT was generally acceptable (Table 2). There was no therapyrelated death.

Ten patients in CR1, who did not undergo ASCT, were also analyzed as control group (Table 1). Their survival time was calculated starting from the completion of the 8 cycles of (R-)CHOP instead of ASCT. When we compared both groups (with and without ASCT) using U-test for distribution of age, serum $\mathrm{LDH}$, interleukin-2 receptor (IL-2R) at presentation and chi-square test for proportion of the presence of B-symptom, extranodal lesion, risk classification by IPI, and administration of R, no difference was showed. However, a significantly larger proportion of patients with stage IV was revealed in ASCT group compared with non-ASCT group $(\mathrm{P}<0.05$ by chi-square test). Therefore, we considered that inadequate bias

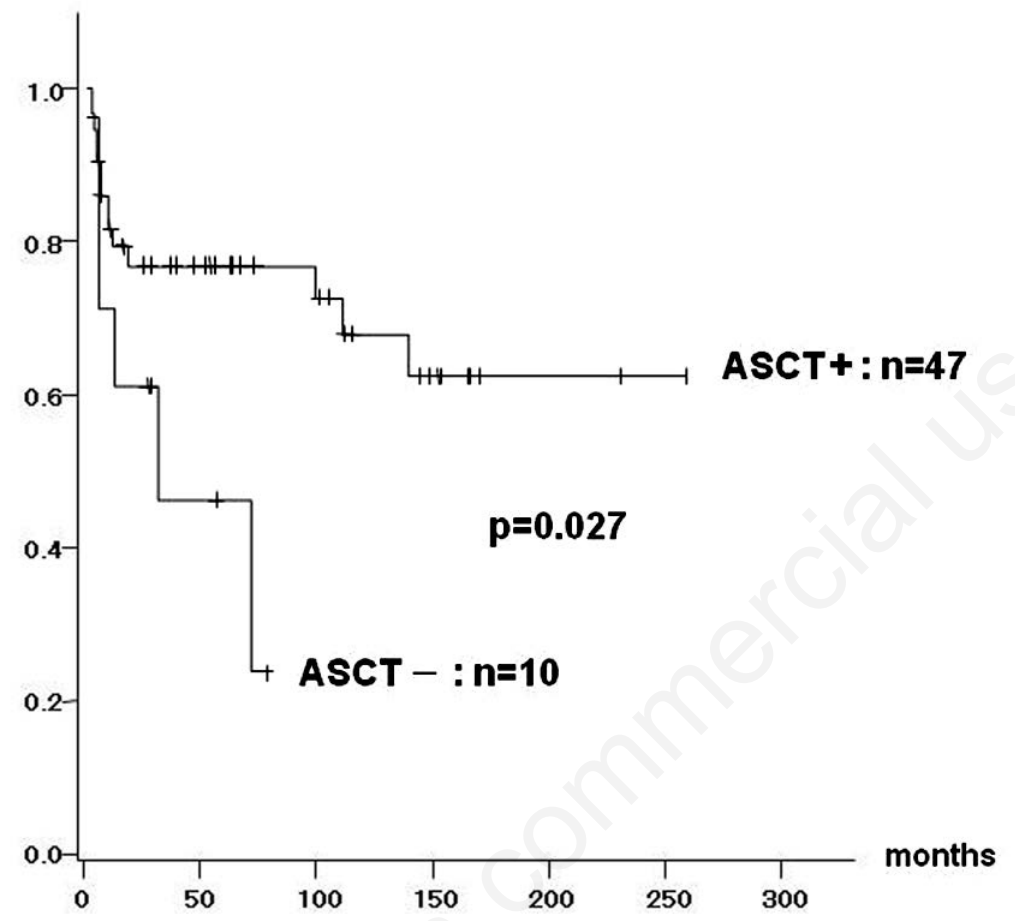

Figure 1. Event free survival of patients with autologous stem cell transplantation (ASCT) compared to those without ASCT. Significant superiority by log-rank test in patients with ASCT is observed $(75.8 \%$ vs $45 \%)$.

Table 1. Patients' characteristics.

\begin{tabular}{lcc} 
& ASCT + & ASCT - \\
Male/Female & $29 / 18$ & $5 / 5$ \\
Age (mean) & $23-69(66.5)$ & $47-68(60.5)$ \\
\hline B symptom & $16(34 \%)$ & $2(20 \%)$ \\
Stage III/IV & $13 / 34$ & $7 / 3$ \\
\hline LDH (mean) & $140-3405(509)$ & $138-874(342)$ \\
Extranodal lesion & $37(78.7 \%)$ & $7(70 \%)$ \\
\hline H-I/H & $29 / 18$ & $7 / 3$ \\
GCB/non-GCB/CD5+/unknown & $7 / 16 / 7 / 17$ & $2 / 6 / 2 /-$ \\
\hline Use of R & $18(38.3 \%)$ & $5(50 \%)$ \\
IL-2R (mean) & $221-29,000(2050)$ & $759-15,000(2050)$ \\
\hline ASCT, autologous stem cell transplantation; H-//H, high-intermediate/high risk according to International Prognostic Index; R, rituximab; GCB, \\
germinal center B-cell type.
\end{tabular}

between the 2 groups was minimized. With a median observation period of 61 months (ranging from 11 to 264), 14 patients of ASCT group relapsed. Their relapse sites was brain in 3 patients, bone marrow in 2 , testis and liver in 1 , respectively. Nodal relapse was seen in the remaining 7. Event-free survival (EFS) of patients who underwent ASCT was 75.8\% after 5 years. This was significantly superior to that of those who did not (45\%, $\mathrm{P}=0.03)$ (Figure 1). Although overall survival (OS) at 5 years tended to be better in ASCT group (79\% vs $52.5 \%$ ), no statistical significance was seen $(\mathrm{P}=0.18)$. Among ASCT group, age difference, normal or elevated LDH value, localized or advanced stage, presence or absence of extranodal lesion, high or high-intermediate risk by IPI, or normal or elevated serum IL-2R did not affect EFS (Table 3). Unexpectedly, use of $R$ was associated with a tendency of shorter sur-

Table 2. Adverse events related to autologous stem cell transplantation.

\begin{tabular}{|c|c|}
\hline Event & N. $(\%)$ \\
\hline Therapy-related death & 0 \\
\hline Febrile neutropenia & $35(74.5)$ \\
\hline MRSA sepsis & $1(2.1)$ \\
\hline Fungemia & $1(2.1)$ \\
\hline CMV antigenemia & $1(2.1)$ \\
\hline Duodenal ulcer & $1(2.1)$ \\
\hline
\end{tabular}

Table 3. Univariate analysis for 5-year event free survival in patients with autologous stem cell transplantation.

\begin{tabular}{lccc}
\multicolumn{1}{c}{ Number } & EFS, \% & P-value \\
Age & & & 0.82 \\
$<60$ & 18 & 77.4 & \\
$\geq 60$ & 29 & 74.8 & \\
Stage & & & 0.16 \\
I/II & 3 & 66.7 & \\
III//V & 44 & 76.4 & \\
\hline LDH & & & 0.88 \\
N & 11 & 78.8 & \\
E & 36 & 73.4 & \\
Extranodal lesion & & & 0.23 \\
Yes & 37 & 70 & \\
None & 10 & 77.6 & \\
\hline IPI & & 0.99 & \\
H & 18 & 79 & \\
H-I & 29 & 70.1 & \\
Serum IL-2R & & & 0.24 \\
N & 13 & 100 & \\
E & 34 & 58.2 & \\
\hline Use of Rituximab & & 0.05 \\
Yes & 18 & 58.2 & \\
No & 29 & 85.7 & \\
\hline
\end{tabular}

$\mathrm{N}$, normal range; $\mathrm{E}$, elevated; H-l/H, high-intermediate/high risk according to International Prognostic Index. 
vival (5-year OS $80.5 \%$ vs $67.6 \%$; $\mathrm{P}=0.25$, EFS $80.5 \%$ vs $58.4 \%, \mathrm{P}=0.08)$.

\section{Discussion}

Progression free survival rate of patients with higher risk group DLBCL is unsatisfactory even in the R-era. ${ }^{16}$ The majority of them experience relapse and the cure is hardly acquired. Although definite usefulness of high-dose therapy followed by ASCT is limited to cases with chemosensitive relapse, ${ }^{15}$ Caballero et al. demonstrated significantly superior OS and EFS of ASCT for DLBCL in CR1 to those in second CR or refractory diseases. ${ }^{10}$ It is suggested that high-dose therapy eliminates minimally resided lesion in clinical CR state. Controversial results are also found in randomized trials. ${ }^{4,5,9,11}$ However, the latter studies include some concerns. Application of various chemotherapy regimens or dose intensity introduced in those studies disturbs to evaluate therapeutic outcomes. Moreover, uneven risk assessment of patients among each study is not capable of precise indication of ASCT. It is particularly unfavorable that the number of induction chemotherapy cycles before ASCT in those studies is too small. ${ }^{9,11}$ On the contrary, studies showing encouraging data contain full course induction, ${ }^{7,8}$ or additional consolidation therapy following induction. ${ }^{12}$ Since the dose intensity of preceding chemotherapy to ASCT might lead to deeper remission, benefit of upfront ASCT as post-remission consolidation might be enhanced.

We therefore analyzed outcome of upfront ASCT employing six courses of (R-)CHOP followed by high-dose chemotherapy to examine whether it prolongs survival in patients with DLBCL classified as higher risk. Consequently, significant superiority in EFS of patients of ASCT group to those without ASCT was observed, although the former included significantly larger number of patients with stage IV than the latter. Despite variant histological subtypes included in our study, influence on their prognosis was unclear because of too small numbers of patients with respective subtypes. We consider that any bias is not contained in our study. It is notable that a considerable number of patients with ASCT (13 of 47, $27.7 \%$ ) have uneventfully survived more than 10 years.

In the last decade, introduction of $\mathrm{R}$ has apparently improved prognosis of DLBCL. ${ }^{22,23}$ $\mathrm{R}$ also appears to purge contaminated lymphoma cells in harvested stem cells to prevent dissemination when transplanted. Feasibility, safety, or survival benefit of the addition of $\mathrm{R}$ to ASCT was actually demonstrated, ${ }^{24-28}$ although our present study failed to prove it. That is probably because relatively frequent brain relapse was occurred in patients who received $\mathrm{R}$ (3 of $19,15.8 \%$ vs 0 of $28,0 \%$ ). Poor delivery of $\mathrm{R}$ to central nervous system should be overcome.

Controversy about indication of ASCT partly depends on enhanced toxicity. Increase of adverse event by high-dose therapy is an important matter of concern. Kameoka et al. observed sinusoidal obstructive syndrome (SOS) in 16 patients out of 30 who received ASCT with identical conditioning regimen as ours. ${ }^{28}$ However, there was no SOS in our study. Accurate frequency of such severe complication of ASCT should be clarified. Hematological or non-hematological adverse events were generally acceptable.

\section{Conclusions}

We thus conclude that upfront ASCT is well tolerable and safe and contributes to survival benefit of higher risk DLBCL, supporting previously described positive data. ${ }^{4,12,25,27}$ Prospective study including a larger number of patients is required to establish appropriate candidates for upfront ASCT among patients with higher risk DLBCL.

\section{References}

1. Vaidya R, Witzig TE. Prognostic factors for diffuse large B-cell lymphoma in the R(x)CHOP era. Ann Oncol 2014;25:212433 .

2. The International Non-Hodgkin's Lymphoma Prognostic Factors Project. A predictive model for aggressive nonHodgkin's lymphoma. N Engl J Med 1993;329:987-94.

3. The Non-Hodgkin's Lymphoma Classification Project. Effect of age on the characteristics and clinical behavior of non-Hodgkin's lymphoma patients. Ann Oncol 1997;8:973-8.

4. Verdonck LF, van Putten WL, Hagenbeek A, et al. Comparison of CHOP chemotherapy with autologous bone marrow transplantation for slowly responding patients with aggressive non-Hodgkin's lymphoma. N Engl J Med 1995;332:1045-51.

5. Martelli M, Vignetti M, Zinzani PL, et al. High-dose chemotherapy followed by autologous bone marrow versus dexamethasone, cisplatin and cytarabine in aggressive non-Hodgkin's lymphoma with partial response to front-line chemotherapy: a retrospective randomized Italian multicenter study. J Clin Oncol 1996;14:53442.

6. Gianni AM, Bregni M, Siena S, et al. High- dose chemotherapy and autologous bone marrow transplantation compared with MACOP-B in aggressive B-cell lymphoma. N Engl J Med 1997;336:1290-7.

7. Santini G, Salvagno L, Leoni P, et al. VACOP-B versus VACOP-B plus autologous bone marrow transplantation for advanced diffuse non-Hodgkin's lymphoma: results of a prospective randomized trial by the non-Hodgkin's Lymphoma Cooperative Study Group. J Clin Oncol 1998;16:2796802.

8. Haioun C, Lepage E, Gisselbrecht C, et al. Survival benefit of high-dose therapy in poor-risk aggressive non-Hodgkin's lymphoma: final analysis of the prospective LNH87-2 protocol-A Groupe d'Etude des Lymphomes de l'Adulte Study. J Clin Oncol 2000;18:3025-30.

9. Gisselbrecht C, Lepage E, Molina T, et al. Shortened first-line high-dose chemotherapy for patients with poor-prognosis aggressive lymphoma. J Clin Oncol 2002; 20:2472-9.

10. Caballero MD, Perez-Simon JA, Iriondo A, et al. High-dose therapy in diffuse large cell lymphoma: results and prognostic factors in 452 patients from the GEL-TAMO Spanish Cooperative Group. Ann Oncol 2003;14:140-51.

11. Martelli M, Gherlinzoni F, De Renzo A, et al. Early autologous stem-cell transplantation versus conventional chemotherapy as front-line therapy in high-risk, aggressive non-Hodgkin's lymphoma: an Italian multicenter randomized trial. J Clin Oncol 2003;21: 1255-62.

12. Milpied N, Deconinck E, Gaillard E, et al. Initial treatment of aggressive lymphoma with high-dose chemotherapy and autologous stem-cell support. N Engl J Med 2004; 350:1287-95.

13. Inano $\mathrm{S}$, Iwasaki $\mathrm{M}$, Iwamoto $\mathrm{Y}$, et al. Impact of high-dose chemotherapy and autologous transplantation as first-line therapy on survival of high-risk diffuse large B cell lymphoma patients: a singlecenter study in Japan. Int J Hematol 2014; 99:162-8.

14. Stiff PJ, Unger JM, Constine LS, et al. Autologous transplantation as consolidation for aggressive non-Hodgkin's lymphoma. N Engl J Med 2013;369:1681-90.

15. Shipp MA, Abeloff MD, Antman KH, et al. International consensus conference on high-dose therapy with hematopoietic stem cell transplantation in aggressive non-Hodgkin's lymphomas: report of the Jury. J Clin Oncol 1999;17:423-9.

16. Gisselbrecht $\mathrm{C}$. Is there any role for transplantation in the rituximab era for diffuse large B-cell lymphoma? Hematology Am Soc Hematol Educ Program 2012;410-6.

17. Caballero MD, Amigo ML, Hernandez JM, 
et al. Alternative mini-BEAM/ESHAP as salvage therapy for refractory nonHodgkin's lymphomas. Ann Hematol 1997;74:79-92.

18. Kaneko H, Shimura K, Fujii H, et al. Feasibility of modified MECP regimen as second-line chemotherapy for refractory or relapsed aggressive non-Hodgkin lymphoma. J Kyoto Pref Univ Med 2011;120:301-10.

19. Cheson BD, Horning SJ, Coiffier B, et al. Report of an International Workshop to standardize response criteria for nonHodgkn's lymphomas. J Clin Oncol 1999;17:1244-53.

20. Juweid ME, Wiseman GA, Vose JM, et al. Response assessment of aggressive nonHodgkin's lymphoma by integrated International Workshop Criteria and fluorine-18-fluorodeoxyglucose positron emission tomography. J Clin Oncol 2005;23: 4652-61.

21. Fischer RI, Gaynor ER, Dahlberg S, et al. Comparison of a standard regimen (CHOP) with three intensive chemotherapy regimens for advanced non-Hodgkin's lymphoma. N Engl J Med 1993;328:1002-6.

22. Coiffier B, Lepage E, Briere J, et al. CHOP chemotherapy plus rituximab compared with CHOP alone in elderly patients with diffuse large B-cell lymphoma. N Engl J Med 2002;346:235-42.

23. Pfreundschuh M, Trumper L, Osterborg A, et al. CHOP-like chemotherapy plus rituximab versus CHOP-like chemotherapy alone in young patients with good-prognosis diffuse large B-cell lymphoma: a randomized controlled trial by the MabThera International Trial (MInT) Group. Lancet Oncol 2006;7:379-91.

24. Han LN, Zhou J, Hirose T, et al. Feasibility and efficacy of high-dose melphalan, cyclophosphamide, etoposide, and dexamethasone (LEED) chemotherapy with or without rituximab followed by autologous stem cell transplantation for aggressive and relapsed non-Hodgkin's lymphoma. Int J Hematol 2006;84:174-81.

25. Fitoussi 0, Belhadj K, Mounier N, et al. Survival impact of rituximab combined with ACVBP and upfront consolidation autotransplantation in high-risk diffuse large B-cell lymphoma for GELA. Haematologica 2011;96:1136-43.

26. Nagle SJ, Woo K, Schuster SJ, et al. Outcomes of patients with relapsed/refractory diffuse large B-cell lymphoma with progression of lymphoma after autologous stem cell transplantation in the rituximab era. Am J Hematol 2013;88:890-4.

27. Takasaki H, Hashimoto C, Fujita A, et al. Upfront autologous stem cell transplantation for untreated high-risk diffuse large B-cell lymphoma in patients up to 60 years of age. Clin Lymphoma Myeloma Leuk 2013;13:404-9.

28. Kameoka Y, Takahashi N, Ishizawa K, et al Safety and feasibility of high-dose ranimustine (MCNU), carboplatin, etoposide, and cyclophosphamide (MCVC) therapy followed by autologous stem cell transplantation for malignant lymphoma. Int $\mathrm{J}$ Hematol 2012;96:624-30. 